\title{
Free of Charge: Analysis of an Urban \\ Collegiate EMS Agency
}

\author{
Cade Herman, EMT-B; Allison Foster, EMT-B; Heather Scianneaux, NREMT-P; \\ Daniel Garrett, MD
}

\section{POSTER PRESENTATION ABSTRACT | ORIGINAL RESEARCH CATEGORY}

Introduction: Tulane Emergency Medical Services (Tulane EMS) is a student-staffed, basic life support (BLS), transporting EMS agency that provides 24/7, free-of-charge service to students, staff, and non-affiliates. We sought to identify the cost savings to Tulane students of activating Tulane EMS by estimating what patients would be charged if New Orleans EMS, the municipal advanced life support (ALS) service that would otherwise respond to on-campus calls, were activated instead. Tulane EMS provides free ground transport to local hospitals for students, staff, and non-affiliates.

Methods: Between August and December 2018, Tulane EMS responded to 325 activations. After excluding all cancellations and ALS transfers, a sample size of 267 was achieved. Per Tulane EMS protocol, a billing itemization form is completed after transfer of care to the emergency department, capturing baseline maintenance fees, applied interventions, equipment use, and transportation decision with a per-mile rate. Alcohol- or substance- related calls were identified on the form. To estimate what New Orleans EMS would bill for comparable service, itemized, line-item billing charges were obtained from a New Orleans EMS representative. To estimate what insured-patients would be charged, Tulane Student Health Insurance (T-SHIP) plan was utilized as the minimum coverage requirement for all students per university policy. Per T-SHIP, ambulance transports are out-of-network services which require meeting a $\$ 500$ deductible and $90 \%$ of remaining charges deemed to be "Usual and Customary" are covered; however, any alcohol- or substance-related transports are not covered. "Usual and Customary" charges were approximated as the total estimated amount that would be billed by New Orleans EMS. Using IBM" SPSS ${ }^{\circ}$ Statistics Version 25, hypothetical charges for each call if New Orleans EMS were to provide the recorded services were calculated via summation code, adding all accrued charges over the course of each call.

Results: Over the study period, 33.1\% of Tulane EMS activations were alcohol- or substance related. On average, the estimated, hypothetical charge to a student if New Orleans EMS were to respond is $\$ 1328.43$ ( $\mathrm{SD}=\$ 370.78$, Range $=\$ 130.00-2219.00)$. Per T-SHIP, this charge is the estimated cost to a student if the call was alcohol- or substance-related. If the chief complaint was otherwise, the estimated cost to a student who had not yet met their deductible would be, on average, $\$ 582.84$.

Discussion/Conclusions: Based on our findings, the presence of Tulane EMS may alleviate a significant financial burden from its patients. Several limitations of this study must be acknowledged. Data on patient demographics - including insurance coverage - were not collected, precluding accounting for insurance variability. In addition, billed charges may not always match what an insurance company deems to be "Usual and Customary." Lastly, New Orleans EMS has a larger scope of practice as an ALS agency and may apply different interventions. Notwithstanding these limitations, quantifying the financial impact of a collegiate EMS agency may be useful to demonstrate the organization's value to university administrators.

Author Affiliations: Tulane Emergency Medical Services, Tulane University, New Orleans, LA, USA

Address for Correspondence: Daniel Garrett, MD.

E-mail: dgarret@tulane.edu

Conflicts of Interest/Funding Sources: By the JCEMS Submission Declaration Form, all authors are required to disclose all potential conflicts of interest and funding sources. The author declared that they have no conflicts of interest. The author declared that they did not receive funding to conduct the program or research associated with this work.

Ethical Compliance: The authors attest that the research associated with this abstract was conducted in accordance with the JCEMS Ethics Guidelines.

Submission History: Received January 7, 2019; accepted for presentation and publication February 10, 2019.

Poster Presentation: This abstract was presented as a poster at the Academic Poster Session of the $26^{\text {th }}$ Annual Conference of the National Collegiate Emergency Medical
Services Foundation; February 23, 2019; Pittsburgh, PA, USA. The authors received the Second Place Award for the Best Poster Presentation Competition.

Published Online: December 31, 2019

Published in Print: December 31, 2019 (Volume 2: Supplemental 1)

Reviewer Information: In accordance with JCEMS editorial policy, poster presentation abstracts undergo double-blind peer-review by at least two reviewers (JCEMS Editorial Board members and/or independent reviewers) prior to acceptance for presentation and publication. JCEMS thanks the anonymous reviewers who contributed to the review of this work.

Copyright: (C) 2019 Herman, Foster, Scianneaux \& Garrett. This is an open access abstract distributed under the terms of the Creative Commons Attribution 4.0 International (CC BY 4.0) License, which permits unrestricted use, distribution, and reproduction in any medium, provided the original author and source are credited. The full license is available at: https://creativecommons.org/licenses/by/4.0/ Electronic Link: https://doi.org/10.30542/JCEMS.2019.02.S1.02 